\title{
LINEAR FUNCTIONALS ON CERTAIN BANACH SPACES
}

\section{E. J. MCSHANE}

1. Introduction. The purpose of this note is to present what is believed to be a simple proof of a theorem on the representation of linear functionals on spaces $L_{p}(p>1)$ and some generalizations of such spaces. Instead of making use of the Radon-Nikodym theorem, the proof utilizes some simple consequences of uniform convexity, and applies at once to spaces $L_{p}$ over arbitrary sets $X$ on a family of whose subsets a measure function is defined, no decomposition of the space being involved. The proofs are stated for complex Banach spaces; the real case allows some obvious simplifications.

\section{Linear functionals and derivatives of norms.}

Lemma 1. Let $g$ be an element of a complex Banach space $B$ and $L a$ linear continuous functional such that $L(g)=|L| \cdot\|g\|$. Then for each $f$ in $B$ we have

$$
|L| D^{-}(\|g+t f\|)_{t=0} \leqq R L(f) \leqq|L| D_{+}(\|g+t f\|)_{t=0} .
$$

If $|L|$ or $\|g\|$ is 0 , this is trivial. If neither of these is 0 , we find that there is no loss of generality-in assuming that $|L|=\|g\|=1$; in fact, we shall apply the lemma only to cases in which $\|g\|=1$. For all complex $z$ and all $f$ in $B$ we have $L(g+z[f-L(f) g])=L(g)=1=|L|$, so

$$
\|g+z[f-L(f) g]\| \geqq 1 \quad \text { for all complex } z \text {. }
$$

The equation

$$
g+t f=(1+t L(f))\left\{g+t[1+t L(f)]^{-1}(f-L(f) g)\right\}
$$

is an identity for $t \neq-1 / L(f)$, and by (2) the norm of the vector in braces is never less than 1 , which is its value at $t=0$. Hence for all real $t$ near 0 we have

$$
\|g+t f\|-\|g\| \geqq|1+t L(f)|-1 \geqq t(R L(f)) .
$$

Dividing by negative $t$ and letting $t$ approach zero yields the first of inequalities (1); with positive $t$ we obtain the other inequality.

Lemma 2. Let $g$ be a nonzero element of a complex Banach space $B$, and let $L$ be a linear continuous functional on $B$ such that $L(g)=|L|$ - $\|g\|$. Assume that for each $f$ in $B$ the function defined for all real $t$ by 1949.

Presented to the Society, September 2, 1949; received by the editors March 30, 
the expression $\|g+t f\|$ is differentiable at $t=0$. Then for all $f$ the equation

$$
L(f)=|L| \frac{d}{d t}[\|g+t f\|+i\|g-i t f\|]_{t=0}
$$

is satisfied.

Under the present hypotheses, the conclusion of Lemma 1 becomes an equation. Writing this for $f$ and for -if, we obtain

$$
\begin{aligned}
& R L(f)=|L| \frac{d}{d t}\|g+t f\|_{t=0}, \\
& \Im L(f)=R L(-i f)=|L| \frac{d}{d t}\|g-i t f\|_{t=0},
\end{aligned}
$$

establishing (3).

Next we recall $[1, \text { p. 396 }]^{1}$ that the space $B$ is uniformly convex if to each positive $\epsilon$ there corresponds a positive $\delta(\epsilon)$ such that if $\|f\|=\|g\|=1$ and $\|f-g\| \geqq \epsilon$, then

$$
\|(f+g) / 2\| \leqq 1-\delta(\epsilon) .
$$

Lemma 3. If $B$ is uniformly convex, to each linear continuous functional $L$ on $B$ there corresponds a unit vector $g_{L}$ such that

$$
|L| D^{-}\left\|g_{L}+t f\right\| \leqq R L(f) \leqq|L| D_{+}\left\|g_{L}+t f\right\|
$$

for all $f$ in $B$. In particular, if $\left\|g_{L}+t f\right\|$ is a differentiable function of $t$ at $t=0$ for all $f$ in $B$, the equation

$$
L(f)=|L| \frac{d}{d t}\left(\left\|g_{L}+t f\right\|+i\left\|g_{L}-i t f\right\|\right)_{t=0}
$$

holds for all $f$ in $B$.

Choose a sequence of unit vectors $f_{1}, f_{2}, \cdots$ such that $L\left(f_{n}\right)$ tends to $|L|$. Let $\epsilon$ be positive; then so is $\delta(\epsilon)$, and for all large $m$ and $n$ we have

$$
L\left(\left[f_{m}+f_{n}\right] / 2\right)=\left[L\left(f_{m}\right)+L\left(f_{n}\right)\right] / 2>|L|[1-\delta(\epsilon)],
$$

so that $\left\|\left(f_{m}+f_{n}\right) / 2\right\|>1-\delta(\epsilon)$. By the definition of uniform convexity, this implies that $\left\|f_{m}-f_{n}\right\|<\epsilon$, and the sequence converges. Let $g_{L}$ be its limit; this is a unit vector. Also

$$
L\left(g_{L}\right)=|L|=|L| \cdot\left\|g_{L}\right\|,
$$

1 Numbers in brackets refer to the bibliography at the end of the paper. 
so the hypotheses of Lemma 1 are satisfied, and the conclusion follows from Lemmas 1 and 2 .

3. Spaces $L_{p}\left(B_{1}\right)$. Now let $m$ be a non-negative measure function on a $\sigma$-field of subsets of a space $X$. A function $f$ on $X$ to a Banach space $B_{1}$ is said to belong to the space $L_{p}\left(B_{1}\right)$ if $\|f(\cdot)\|_{1}^{p-1} f(\cdot)$ is summable in the sense of Bochner over $X$, where $\|\cdot\|_{1}$ denotes the norm in the space $B_{1}$. (If the reader is interested only in the usual space $L_{p}$, he should interpret $B_{1}$ as the real or complex number system, $\|\cdot\|_{1}$ as absolute value, and the integral as the Lebesgue integral.) Assume $p>1$ and define as usual $p^{\prime}=p /(p-1)$. It is then known that if $B_{1}$ is uniformly convex (in particular, if it is the real or complex number system), $L_{p}\left(B_{1}\right)$ is also uniformly convex $[2$, p. 504]; we append another proof of this fact to the present note.

THEOREM I. Let $B_{1}$ be a uniformly convex Banach space such that for each nonzero $g$ in $B_{1}$ and each $f$ in $B_{1}$, the function $=\|g+t f\|_{1}$ is differentiable at $t=0$. Let $p$ be greater than 1 , and let $L$ be a functional linear and continuous on $L_{p}\left(B_{1}\right)$. Then there exists a unit vector $g_{L}$ in $L_{p}\left(B_{1}\right)$ such that for every $f$ in $L_{p}\left(B_{1}\right)$ we have

$$
\begin{aligned}
L(f)=|L| \int_{X}\left\|g_{L}(x)\right\|_{1}^{p-1} \frac{d}{d t}\left[\| g_{L}(x)\right. & +t f(x) \|_{1} \\
& \left.+i\left\|g_{L}(x)-i t f(x)\right\|_{1}\right]_{t=0} m(d x),
\end{aligned}
$$

wherein the derivative in the integrand is to be assigned any arbitrary finite value when the factor multiplying it has the value 0.

Let $g_{L}$ be the unit vector in $L_{p}\left(B_{1}\right)$ whose existence is guaranteed by Lemma 3 , and let $f$ belong to $L_{p}\left(B_{1}\right)$. The equation

$$
\frac{d}{d t}\left\|g_{L}(x)+t f(x)\right\|_{1}^{p}=p\left\|g_{L}(x)+t f(x)\right\|_{1}^{p-1} \frac{d}{d t}\left\|g_{L}(x)+t f(x)\right\|_{1}
$$

holds whenever $g_{L}(x)+t f(x) \neq 0$ by elementary differentiation, and when that vector is 0 it continues to hold if the derivative in the right member is assigned any finite value. For $|t|<1$ the right member cannot exceed the function $p\left[\left\|g_{L}(x)\right\|_{1}+\|f(x)\|_{1}\right]^{p}$, which is summable. This permits us to differentiate under the integral sign in the integral which defines $\left\|g_{L}+t f\right\|$; recalling that $\left\|g_{L}\right\|=1$, we obtain

(7) $\frac{d}{d t}\left\|g_{L}+t f\right\|_{t=0}=\int_{X}\left\|g_{L}(x)\right\|_{1}^{p-1}\left[\frac{d}{d t}\left\|g_{L}(x)+t f(x)\right\|_{1}\right]_{L=0} m(d x)$.

Thus the hypotheses of Lemma 2 are satisfied, and the conclusion of 
Lemma 2 is equation (6).

As a special case, we obtain the representation of the general linear continuous functional on complex $L_{p}$.

TheOREM II. Let $L$ be a continuous linear functional on the complex Banach space $L_{p}$ of functions on $X$, wherein $p>1$. Then there exists an element $g$ of $L_{p^{\prime}}$, where $p^{\prime}=p /(p-1)$, such that the norm of $g$ in $L_{p^{\prime}}$ is $|L|$ and

$$
L(f)=\int_{X} g(x) f(x) m(d x)
$$

for all $f$ in $L_{p}$.

If $a$ and $b$ are complex numbers such that $a \neq 0$, from

$$
\begin{aligned}
|a+t b| & =|a|[|1+t(b / a)|] \\
& =|a|\left[(1+R t b / a)^{2}+(J t b / a)^{2}\right]^{1 / 2}
\end{aligned}
$$

we obtain

$$
[d|a+t b| / d t]_{t=0}=|a| R(b / a)=R \bar{a} b /|a|,
$$

whence

$$
\begin{aligned}
{[d|a+t b| / d t+i d|a-i t b| / d t]_{t=0} } \\
=R[\bar{a} b /|a|]+i \Im[\bar{a} b /|a|]=\bar{a} b /|a| .
\end{aligned}
$$

Thus the integrand in (6) takes the form

$$
\left\|g_{L}(x)\right\| \overline{p-2} \overline{g_{L}(x)} f(x)
$$

whenever $g_{L}(x) \neq 0$. If we now define

$$
\begin{array}{ll}
g(x)=|L| \cdot\left\|g_{L}(x)\right\| p-\overline{g_{L}(x)} & \text { where } \quad g_{L}(x) \neq 0, \\
g(x)=0 & \text { wherever } g_{L}(x)=0,
\end{array}
$$

we see that (8) holds. Now $g(x)$ is everywhere a non-negative multiple of the conjugate of $g_{L}(x)$, and by the definition of $p^{\prime}$ we have

$$
|g(x)|^{p^{\prime}}=|L|^{p^{\prime}}\left|g_{L}(x)\right|^{p} .
$$

Hence $g$ is in $L_{p^{\prime}}$, and its norm in $L_{p^{\prime}}$ is $|L|$, completing the proof.

THEOREM III. Let $\mu$ be a measure function on a $\sigma$-field of subsets of $a$ set $Y$, and let $m$ be a measure function on a $\sigma$-field of subsets of $a$ set $X$. Let $L_{q}$ be the space of complex functions on $Y$ such that $\left.|f(y)|\right|^{q-1} f(y)$ is summable over $Y$, and $L_{p}\left(L_{q}\right)$ the space of functions on $X$ to $L_{q}$ such that $\|f(x)\|_{a}^{p-1} f(x)$ is Bochner summable over $X$, the symbol $\|\cdot\|_{q}^{q}$ denoting 
the norm in $L_{q}$. Assume $p$ and $q$ greater than 1 , and define $p^{\prime}=p /(p-1)$, $q^{\prime}=q /(q-1)$. Let $L$ be a linear continuous functional on the complex Banach space $L_{p}\left(L_{q}\right)$. Then there exists an element $g$ of $L_{p^{\prime}}\left(L_{q^{\prime}}\right)$, with norm $|L|$ in that space, such that for every element

$$
f=(f(x) \mid x \in X)=((f(y, x) \mid y \in Y) \mid x \in X)
$$

of the space $L_{p}\left(L_{q}\right)$ we have

$$
L(f)=\int_{X}\left\{\int_{Y} g(y, x) f(y, x) \mu(d y)\right\} m(d x) .
$$

For $L$ we first use equation (6), recalling that for each $x$ the value of $g_{L}(x)$ is the function $\left(g_{L}(y, x) \mid y \in Y\right)$. For the derivative in the integrand of (6) we substitute from (7), applied to the space $Y$ instead of $X$. The inner integrand will contain a derivative, which we replace by its value as computed in (9). The result is

$$
L(f)=|L| \int_{X}\left\{\left\|g_{L}(x)\right\|^{p-1} \int_{Y}\left|g_{L}(y, x)\right|^{q-2} \overline{g_{L}(y, x)} f(y, x) \mu d y\right\} m(d x),
$$

where the inner integrand is to be understood to mean 0 whenever $g_{L}(y, x)$ vanishes. We define

$$
g(y, x)=|L||| g_{L}(x)||^{p-1}\left|g_{L}(y, x)\right|^{q-2} \overline{g_{L}(y, x)}
$$

wherever $g_{L}(y, x) \neq 0$, and set $g(y, x)=0$ where $g_{L}(y, x)=0$. Then (10) holds. The verification of the other statements about $g$ is much the same as in the proof of Theorem II.

In Theorem II we extended the representation theorem for linear functionals on $L_{p}$ to maximum generality as regards the space $X$. It is not possible to extend the theorem on the conjugate space of $L_{1}$ to a corresponding generality, as is shown by the following example, which is the result of a conversation with T. A. Botts and V. L. Klee. Let $X$ consist of the points of the Euclidean plane. Let the exterior measure of a countable set $E$ be 1 or 0 according as the origin is or is not in $E$; the exterior measure of every uncountable set is $+\infty$. This exterior measure generates a Carathéodory measure which coincides with itself, all sets being measurable. A function $f$ is summable if and only if it is zero except on a countable set and $f(0)$ is finite, and in this case its integral is $f(0)$. All spaces $L_{p}(p \geqq 1)$ coincide, and the norm is $\|f(\cdot)\|=|f(0)|$, and all are conjugate to each other. But the space conjugate to $L_{1}$ is $L_{1}$, which is distinct from the space of essentially measurable functions on $X$.

4. Proof of uniform convexity of spaces $L_{p}(B)$. We now prove that 
when $L_{p}(B)$ is defined as above, and $B$ is uniformly convex and $p>1$, the space $L_{p}(B)$ is also uniformly convex. For the norms in $B$ and $L_{p}(B)$ we shall use the symbols $\|\cdot\|,\|\cdot\|_{p}$ respectively. As a first step, we show that to each positive $\epsilon$ corresponds a positive $\delta(\epsilon)$ such that if $f$ and $g$ are in $B$, and $1=\|f\| \geqq \| g$, and $\|f-g\| \geqq \epsilon$, then

$$
\|(f+g) / 2\| p \leqq[1-\delta(\epsilon)]\left[\|f\|^{p}+\|g\|^{p}\right] / 2 .
$$

Assume this false; then there exist $\epsilon>0$ and sequences $f_{n}, g_{n}$ of elements of $B$ such that $1=\left\|f_{n}\right\| \geqq\left\|g_{n}\right\|,\left\|f_{n}-g_{n}\right\| \geqq \epsilon$, and

$$
\lim _{n \rightarrow \infty} \frac{\left\|\left(f_{n}+g_{n}\right) / 2\right\|^{p}}{\left[\left\|f_{n}\right\|^{p}+\left\|g_{n}\right\|^{p}\right] / 2}=1 \text {. }
$$

From the convexity of $t^{p}(0 \leqq t \leqq 1)$ we obtain

$$
([1+t] / 2)^{p}<\left(1+t^{p}\right) / 2 \quad \text { for } 0 \leqq t<1
$$

so by (12) and the triangle inequality

$$
\lim _{n \rightarrow \infty} \frac{\left(1+\left\|g_{n}\right\|\right)^{p 2^{p-1}}}{1+\left\|g_{n}\right\|^{p}}=1 .
$$

But by (13) this implies $\lim \left\|g_{n}\right\|_{1}=1$. Define $u_{n}=g_{n} /\left\|g_{n}\right\|$; then $\lim \left\|u_{n}-g_{n}\right\|=0$, so $\lim \inf \left\|u_{n}-f_{n}\right\|=\lim$ inf $\left\|g_{n}-f_{n}\right\| \geqq \epsilon$, and by (12)

$$
\lim \left\|\left(f_{n}+u_{n}\right) / 2\right\|=1 .
$$

This contradicts the uniform convexity of $B$.

As a corollary, if $f$ and $g$ are not both 0 we have

$$
\begin{aligned}
& \|(f+g) / 2\|^{p} \\
& \quad \leqq[1-\delta(\|f-g\| / \sup (\|f\|,\|g\|))]\left[\|f\|^{p}+\|g\|^{p}\right] / 2 .
\end{aligned}
$$

Now assume that $f(\cdot)$ and $g(\cdot)$ are in $L_{p}(B)$, and that $\epsilon$ is positive, and that $\|f\|=\|g\|=1$ and $\|f-g\|_{p} \geqq \epsilon$. Let $E$ be the subset of $X$ on which

$$
\begin{aligned}
\|f(x)-g(x)\|^{p} & \geqq\left(\epsilon^{p} / 4\right)\left(\|f(x)\|^{p}+\|g(x)\|^{p}\right) \\
& \geqq\left(\epsilon^{p} / 4\right) \sup \left(\|f(x)\| p,\left\|g^{p}(x)\right\|\right) .
\end{aligned}
$$

On this set we have, by (14)

$$
\|[f(x)+g(x)] / 2\|^{p} \leqq\left[1-\delta\left(\epsilon / 4^{1 / p}\right)\right]\left[\|f(x)\|^{p}+\|g(x)\| p\right] / 2 .
$$

The integral of $\|f-g\|^{p}$ over $X-E$ is at most $\epsilon^{p} / 2$, so

$$
\int_{E}\|f(x)-g(x)\| \|^{p} m(d x) \geqq \epsilon^{p} / 2 .
$$


Thus the functions which coincide with $f(\cdot)$ and $g(\cdot)$ on $E$ and vanish on $X-E$ have distance at least $\epsilon / 2^{1 / P}$. So at least one of them has distance not less than $\epsilon / 2 \cdot 2^{1 / p}$ from the origin, and

$$
\sup \left(\int_{E}\|f(x)\|^{p_{m}}(d x), \quad \int_{E}\|g(x)\| p_{m}(d x)\right) \geqq \epsilon^{p / 2^{p+1}} .
$$

Now by (16) and (18),

$$
\begin{aligned}
& \int_{X}\left[\left(\|f(x)\|^{p}+\|g(x)\|^{p}\right) / 2\right] m(d x)-\int_{X}\left[\|(f(x)+g(x) \| / 2]^{p} m(d x)\right. \\
& \geqq \int_{E}\left[\left(\|f(x)\|^{p}+\|g(x)\|^{p}\right) / 2\right] m(d x)-\int_{E}[\|f(x)+g(x)\| / 2]^{p} m(d x) \\
& \geqq \int_{E} \delta\left(\epsilon / 4^{1 / p}\right)\left\{\left[\|f(x)\|^{p}+\|g(x)\|^{p}\right] / 2\right\} m(d x) \\
& \geqq \delta\left(\epsilon / 4^{1 / p}\right)\left(\epsilon^{p} / 2^{p+2}\right),
\end{aligned}
$$

whence

$$
\|f(\cdot)+g(\cdot)\|_{p} \leqq\left[1-\delta\left(\epsilon / 4^{1 / p}\right)\left(\epsilon^{p / 2^{p+2}}\right)\right]^{1 / p} .
$$

If we define $\delta_{1}(\epsilon)$ to be the difference between 1 and the right member of (19), we see that $\delta_{1}(\epsilon)>0$, and $L_{p}(B)$ is uniformly convex.

\section{BIBLIOGRAPHY}

1. J. A. Clarkson, Uniformly convex spaces, Trans. Amer. Math. Soc. vol. 40 (1936) pp. 396-414.

2. M. M. Day, Some more uniformly convex spaces, Bull. Amer. Math. Soc. vol. 47 (1941) pp. 504-507.

UNIVERSITY OF VIRGINIA 